\title{
Weniger Exazerbationen beim „Eosinophilie-Phänotyp“
}

\author{
Bei COPD-Patienten mit Eosinophilie, die unter einer \\ ICS-einschließenden Dreifachtherapie weiterhin \\ symptomatisch bleiben und häufige Exazerbationen \\ erleiden, kann eine Therapie mit dem IL-5-Antikörper \\ Mepolizumab die Exazerbationsfrequenz reduzieren.
}

Beim ERS-Kongress 2017 Mailand und gleichzeitig im „New England Journal of Medicine" wurden die Ergebnisse der zwei Phase 3-Doppelblindstudien METREX und METREO vorgestellt. Untersucht wurde in einem reichlich komplexen Studiendesign der Wert einer IL-5-Blockade mit Mepolizumab bei Patienten mit schwerer COPD.

Wie Studienleiter Prof. Frank Sciurba von der University in Pittsburgh darlegte, empfehlen die aktuellen GOLD-Leitlinien eine Triple-Therapie aus ICS/LABA/LAMA, wenn der COPDPatient sonst nicht symptomfrei wird. Etwa ein Drittel der Patienten bleibt auch unter Dreifachtherapie symptomatisch. $40 \%$ dieser Patienten würden einen „eosinophilen Phänotyp" aufweisen, erkennbar an Eosinophilen-Zahlen über 150-200 Zellen/ $\mu \mathrm{l}$.

Mepolizumab reduziert die Eosinophilenzahlen. Es ist wirksam in der Therapie des schweren eosinophilen Asthmas. So lag es nahe, den Wert des Antikörpers auch bei schwerer eosinophiler COPD zu testen.

Für die Studien wurden insgesamt etwa 1.500 Patienten rekrutiert. Alle wurden bereits mit einer Triple-Therapie behandelt, $95 \%$ befanden sich im GOLD-Stadium D, der CAT-Wert lag im Schnitt bei 19. In den vergangenen 12 Monaten hatten die Patienten im Schnitt etwa 2,5 moderate oder schwere Exazer-

bationen erlitten. Die Postbronchodilatator-FEV ${ }_{1}$ lag bei ca. 1,25 1, die FEV $\mathrm{F}_{1}$ bei ca. $45 \% .25 \%$ der Patienten waren aktuelle Raucher.

\section{Wirrwarr bzgl. der Eosinophilen-Einschluss-Werte}

Die Eosinophilen-Zahlen unterschieden sich in einzelnen Gruppen. In der Gruppe vom „Eosinophilen-Phänotyp“ der METREX-Studie hatten $94 \%$ der Patienten Werte über $150 / \mathrm{mm}^{3}$, in der METREO-Studie waren dies etwa $80 \%$, im METREX-Gesamtkollektiv etwa die Hälfte der Patienten.

Mepolizumab (Nucala ${ }^{\circledR}$ ) wurde alle vier Wochen in einer Dosis von $100 \mathrm{mg}$ (METREX) bzw. $100 \mathrm{mg}$ oder $300 \mathrm{mg}$ (METREO) verabreicht.

\section{Weniger mittelschwere Exazerbationen beim Eosinophilen-Phänotyp}

Primärer Endpunkt war die jährliche Rate moderater bzw. schwerer Exazerbationen. Eine signifikante Differenz fand sich diesbezüglich in der Kohorte der METREX-Studie, die einen eosinophilen Phänotyp aufwies: Im Mittel 1,4 Exazerbationen unter Mepolizumab gegenüber 1,71 unter Placebo (RR: 0,82, $\mathrm{p}=0,04)$.

Im METREX-Gesamtkollektiv fand sich kein Unterschied bei den Exazerbationen. In der METREO-Studie erreichten die Unterschiede in den beiden Dosierungsgruppen jeweils keine statistische Signifikanz.

Die Autoren arbeiteten mithilfe präspezifizierter Metaanalysen beider Studien heraus, dass der Nutzen einer MepolizumabBehandlung umso größer war, je höher die Eosinophilen-Werte waren. Dies hätte man angesichts der Asthma-Daten auch schon vorher wissen können. Man fragt sich, warum die Autoren nicht vorab klare Schwellenwerte für die Eosinophilenzahlen im gesamten Studienkollektiv festgelegt haben.

Für Studienleiter Sciurba „demonstriert die Studie das Potenzial von Mepolizumab, COPD-Exazerbationen zu reduzieren
METREX und METREO waren zwei wichtige Studien, die beim ERS-Kongress vorgestellt wurden. Demnach scheint ein IL-5-Antikörper bei schwerer COPD Vorteile zu bringen. bei Patienten, die bereits eine optimale COPD-Erhaltungstherapie haben, sofern die Eosinophilenwerte als Biomarker eingesetzt werden."

\section{Keine Wirksamkeit auf Symptomatik oder Lebensqualität}

Die beobachtete Reduktion der Exazerbationen war mit einer Differenz von 0,3 pro Jahr nicht sehr eindrücklich. Schwere Exazerbationen, die zu Behandlung in der Notaufnahme oder im Krankenhaus führten, waren im Studienkollektiv selten und unter Mepolizumab in der Tendenz eher häufiger (0,3 vs. 0,26 pro Jahr). Auf die Symptomatik (CAT-Score) oder die Lebensqualität hatte Mepolizumab keinen signifikanten positiven Einfluss.

Dr. med. Dirk Einecke

Quellen: European Respiratory Society Congress 2017, Mailand, 9.-13.9.2017; Pavord ID, et al. Mepolizumab for Eosinophilic Chronic Obstructive Pulmonary Disease. N Engl J Med 2017, doi: 10.1056/NEJMoa1708208 